\title{
On New Weighted Ostrowski Type inequalities Involving Integral Means over End Intervals and Application
}

\author{
A. Qayyum ${ }^{1}$, M. Shoaib ${ }^{2}$, I. Faye S,* $^{1, *}$ \\ ${ }^{1}$ Department of Fundamental and Applied Sciences, Universiti Teknologi Petronas, Malaysia \\ ${ }^{2}$ Department of Mathematics, University of Hail, 2440, Saudi Arabia \\ *Corresponding author: ibrahima_faye@petronas.com.my
}

Received October 10, 2014; Revised February 08, 2015; Accepted May 20, 2015

\begin{abstract}
The aim of this paper is to establish new inequalities using weight function which generalizes the inequalities of Dragomir, Wang and Cerone. In this article we obtain bounds for the deviation of a function from a combination of weighted integral means over the end intervals covering the entire interval. A variety of earlier results are recaptured as particular instances of the current development. Applications for cumulative distribution function is also discussed.
\end{abstract}

Keywords: ostrowski inequality, weight function, weighted integral mean

Cite This Article: A. Qayyum, M. Shoaib, and I. Faye, "On New Weighted Ostrowski Type inequalities Involving Integral Means over End Intervals and Application." Turkish Journal of Analysis and Number Theory, vol. 3, no. 2 (2015): 61-67. doi: 10.12691/tjant-3-2-5.

\section{Introduction}

Integration with weight functions is used in many mathematical branches such as: approximation theory, spectral analysis, statistical analysis, theory of distributions and functional analysis. In 1938, Ostrowski [13] proved his famous inequality:

Theorem 1 Let $f:[a, b] \rightarrow \mathbb{R}$ be continuous on $[a, b]$ and differentiable on $(a, b)$, and assume $\left|f^{\prime}(x)\right| \leq M$ for all $x \in(a, b)$, then the inequality holds

$$
|S(f ; a, b)| \leq\left[\frac{1}{4}+\frac{\left(x-\frac{a+b}{2}\right)^{2}}{(b-a)^{2}}\right] \frac{M}{b-a},
$$

for all $x \in[a, b]$. The constant $\frac{1}{4}$ is best possible.

Several generalizations of the above integral inequality are considered by many authors such as $[2,3,4,10,16,17]$.

The functional $S(f ; a, b)$ given below, represents the deviation of $f(x)$ from its integral mean over $[a, b]$

$$
S(f ; a, b)=f(x)-M(f ; a, b),
$$

and

$$
M(f ; a, b)=\frac{1}{b-a} \int_{a}^{b} f(x) d x
$$

Milovanovic' and Pecaric' in [11] gave first generalization of Ostrowski's inequality. Dragomir and Wang [7] introduced Ostrowski-Grüss type inequality. Cheng gave a sharp version of the mentioned inequality in [3]. Dragomir and Wang [5,6,7,8,9] and Cerone [1] pointed out a result to the above. We establish new inequalities, which are more generalized as compared to previous inequalities developed in $[1,5,6,7,8,9]$ and $[14,15]$. Moreover, our results are further generalized by the introduction of weight function.

The approach of Dragomir and Wang [5,6,7,8,9], Cerone [1], and Qayyum et. al [14,15] for obtaining bounds depended on Peano kernel while we use weighted Peano kernel in our findings. This approach not only generalizes the results of Dragomir and Wang $[5,6,7,8,9]$, Cerone [1] and Qayyum [14,15], but also gives some other interesting inequalities as special cases. In the last section, we will apply our main result for cumulative distribution function.

Montgomery's identity states that for absolutely continuous mappings $f:[a, b] \rightarrow \mathbb{R}$

$$
f(x)=\frac{1}{b-a} \int_{a}^{b} f(t) d t+\frac{1}{b-a} \int_{a}^{b} P(x, t) f^{\prime}(t) d t,
$$

where

$$
P(x, t)=\left\{\begin{array}{cc}
t-a & \text { if } a \leq t \leq x \leq b \\
t-b & \text { if } a \leq x<t \leq b .
\end{array}\right.
$$

Dragomir and Wang $[5,6,7,8]$ utilizing an integration by parts argument, obtained

$$
|S(f ; a, b)|
$$




$$
\leq\left\{\begin{array}{c}
\frac{1}{b-a}\left[\left(\frac{b-a}{2}\right)^{2}+\left(x-\frac{a+b}{2}\right)^{2}\right]\left\|f^{\prime}\right\|_{\infty}, f^{\prime} \in L_{\infty}[a, b] \\
\frac{1}{b-a}\left[\frac{(x-a)^{q+1}+(b-x)^{q+1}}{q+1}\right]^{\frac{1}{q}}\left\|f^{\prime}\right\|_{p}, \\
f^{\prime} \in L_{p}[a, b], p>1, \frac{1}{p}+\frac{1}{q}=1 \\
\frac{1}{b-a}\left[\frac{b-a}{2}+\left|x-\frac{a+b}{2}\right|\right]\left\|f^{\prime}\right\|_{1}, f^{\prime} \in L_{1}[a, b] .
\end{array}\right.
$$

where $f:[a, b] \rightarrow \mathbb{R}$ is absolutely continuous on $[a, b]$ and the constants $\frac{1}{4},\left[\frac{1}{q+1}\right]^{\frac{1}{q}}$ and $\frac{1}{2}$ are sharp. $L_{p}$ is defined in [14].

Cerone [1], proved the following inequality:

Lemma 1 Let $f:[a, b] \rightarrow \mathbb{R}$ be absolutely continuous mapping and define

$$
\tau(x ; \alpha, \beta)=f(x)-\frac{1}{\alpha+\beta}\left[\begin{array}{l}
\alpha M(f ; a, x) \\
+\beta M(f ; x, b)
\end{array}\right]
$$

where

$$
M(f ; a, b)=\frac{1}{b-a} \int_{a}^{b} f(x) d x
$$

then

$$
\leq\left\{\begin{array}{l}
\frac{1}{2(\alpha+\beta)}\left[\begin{array}{l}
\alpha(x-a) \\
+\beta(b-x)
\end{array}\right]\left\|f^{\prime}\right\|_{\infty}, f^{\prime} \in L_{\infty}[a, b] \\
\left.\frac{1}{(\alpha+\beta)(q+1) \frac{1}{q}}\left[\begin{array}{l}
\alpha^{q}(x-a) \\
+\beta^{q}(b-x)
\end{array}\right]\right]^{\frac{1}{q}}\left\|f^{\prime}\right\|_{p}, f^{\prime} \in L_{p}[a, b], \\
\frac{1}{2}\left(1+\frac{|\alpha-\beta|}{\alpha+\beta}\right)\left\|f^{\prime}\right\|_{1}, f^{\prime} \in L_{1}[a, b] .
\end{array}\right.
$$

Now we express some generalizations (non weighted version) of cerone's result [1].

Lemma 2 Let $P(x, t):[a, b] \rightarrow \mathbb{R}$, the peano type kernel, is given by

$$
p(x, t, h)=\left\{\begin{array}{l}
\frac{\alpha}{\alpha+\beta} \frac{1}{x-a}\left[t-\left(a+h \frac{b-a}{2}\right)\right], a \leq t \leq x, \\
\frac{\beta}{\alpha+\beta} \frac{1}{b-x}\left[t-\left(b-h \frac{b-a}{2}\right)\right], x<t \leq b,
\end{array}\right.
$$

Then,

$$
\begin{gathered}
|\tau(x ; \alpha, \beta)|=\mid \frac{1}{\alpha+\beta}\left[\begin{array}{c}
\frac{\alpha}{x-a}\left\{x-\left(a+h \frac{b-a}{2}\right)\right\} \\
\left.-\frac{\beta}{b-x}\left\{x-\left(b-h \frac{b-a}{2}\right)\right\}\right] f(x)(9)
\end{array}\right] \\
+\frac{h}{\alpha+\beta}\left(\frac{b-a}{2}\right)\left(\frac{\alpha}{x-a} f(a)+\frac{\beta}{b-x} f(b)\right) \\
-\frac{1}{\alpha+\beta}\left[\frac{\alpha}{x-a} \int_{a}^{x} f(t) d t+\frac{\beta}{b-x} \int_{x}^{b} f(t) d t\right]
\end{gathered}
$$

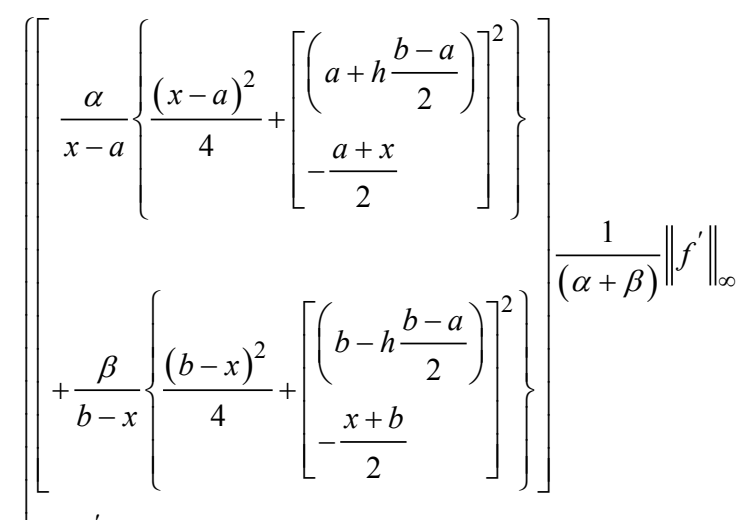$$
f^{\prime} \in L_{\infty}[a, b]
$$$$
\left[\begin{array}{l}
\left.\frac{\alpha^{q}}{(x-a)^{q}}\left\{\begin{array}{l}
\left(x-\left(a+h \frac{b-a}{2}\right)\right)^{q+1} \\
-\left(h \frac{a-b}{2}\right)^{q+1}
\end{array}\right\}\right]^{\frac{1}{q}} \\
+\frac{\beta^{q}}{(b-x)^{q}}\left\{\begin{array}{l}
\left(b-\left(x+h \frac{b-a}{2}\right)\right)^{q+1} \\
-\left(h \frac{a-b}{2}\right)^{q+1}
\end{array}\right\}
\end{array}\right]
$$$$
\times \frac{1}{(q+1)^{\frac{1}{q}}(\alpha+\beta)}\left\|f^{\prime}\right\|_{p} \text {, }
$$$$
f^{\prime} \in L_{p}[a, b],
$$$$
\|\left[\begin{array}{l}
(\alpha+\beta)-h \frac{b-a}{2}\left[\frac{\alpha(b-x)+\beta(x-a)}{(x-a)(b-x)}\right] \\
+\left|(\alpha-\beta)+h \frac{b-a}{2}\left[\frac{\beta(x-a)-\alpha(b-x)}{(x-a)(b-x)}\right]\right| \\
f^{\prime} \in L_{1}[a, b] .
\end{array}\right.
$$

Qayyum et.al [14] proved another non-weighted version. 
Lemma 3 Denote by $P(x,):.[a, b] \rightarrow \mathbb{R}$ the kernel is given by

$$
P(x, t):=\left\{\begin{array}{l}
\frac{\alpha}{2(\alpha+\beta)(x-a)}(t-a)^{2}, a \leq t \leq x, \\
\frac{\beta}{2(\alpha+\beta)(b-x)}(t-b)^{2}, x<t \leq b,
\end{array}\right.
$$

Then,

$$
\begin{aligned}
& |\tau(x ; \alpha, \beta)| \\
& =\mid \frac{1}{2(\alpha+\beta)}[\alpha(x-a)-\beta(b-x)] f^{\prime}(x)-f(x) \\
& +\frac{1}{\alpha+\beta}[\alpha M(f ; a, x)+\beta M(f ; x, b)] \mid \\
& \int\left[\alpha(x-a)^{2}+\beta(b-x)^{2}\right] \frac{\left\|f^{\prime \prime}\right\|_{\infty}}{6(\alpha+\beta)}, f^{\prime \prime} \in L_{\infty}[a, b] \\
& \leq\left\{\begin{array}{l}
\frac{1}{(2 q+1)^{\frac{1}{q}}}\left[\alpha^{q}(x-a)^{q+1}+\beta^{q}(b-x)^{q+1}\right]^{\frac{1}{q}} \frac{\left\|f^{\prime \prime}\right\|_{p}}{2(\alpha+\beta)}, \\
f^{\prime \prime} \in L_{p}[a, b],
\end{array}\right. \\
& (\alpha(x-a)+\beta(b-x)+|\alpha(x-a)-\beta(b-x)|) \frac{\left\|f^{\prime \prime}\right\|_{1}}{4(\alpha+\beta)} \\
& , f^{\prime \prime} \in L_{1}[a, b] \text {. }
\end{aligned}
$$

Qayyum et.al [15] proved a generalized non-weighted version of Cerone's result [1].

Lemma 4 Let $f:[a, b] \rightarrow \mathbb{R}$ be an absolutely continuous mapping. Denote by $P(x,):.[a, b] \rightarrow \mathbb{R}$, the kernel $P(x, t, h)$ is given by

$$
P(x, t, h)=\left\{\begin{array}{l}
\frac{\alpha}{\alpha+\beta} \frac{1}{x-a} \frac{1}{2}\left[t-\left(a+h \frac{b-a}{2}\right)\right]^{2}, a \leq t \leq x, \\
\frac{\beta}{\alpha+\beta} \frac{1}{b-x} \frac{1}{2}\left[t-\left(b-h \frac{b-a}{2}\right)\right]^{2}, x<t \leq b,
\end{array}\right.
$$

Then,

$$
\begin{gathered}
\tau(x ; \alpha, \beta):=\mid \frac{1}{2(\alpha+\beta)}\left[\frac{\alpha}{x-a}\left(x-\left(a+h \frac{b-a}{2}\right)\right)^{2}\right. \\
\left.-\frac{\beta}{b-x}\left(x-\left(b-h \frac{b-a}{2}\right)\right)^{2}\right] f^{\prime}(x)
\end{gathered}
$$

$$
\begin{aligned}
& -\frac{1}{(\alpha+\beta)}\left[\frac{\alpha}{x-a}\left(x-\left(a+h \frac{b-a}{2}\right)\right)\right. \\
& \left.-\frac{\beta}{b-x}\left(x-\left(b-h \frac{b-a}{2}\right)\right)\right] f(x) \\
& -\frac{1}{\alpha+\beta} h \frac{b-a}{2}\left[\frac{\alpha}{x-a} f(a)+\frac{\beta}{b-x} f(b)\right] \\
& +\frac{1}{\alpha+\beta} h^{2} \frac{(b-a)^{2}}{8}\left[\frac{\beta}{b-x} f^{\prime}(b)-\frac{\alpha}{x-a} f^{\prime}(a)\right] \\
& +\frac{1}{\alpha+\beta}[\alpha M(f ; a, x)+\beta M(f ; x, b)] \mid \\
& \left\{\begin{array}{l}
\frac{\alpha}{x-a}\left\{\begin{array}{l}
{\left[x-\left(a+h \frac{b-a}{2}\right)\right]^{3}} \\
+\left(h \frac{b-a}{2}\right)^{3}
\end{array}\right\} \\
-\frac{\beta}{b-x}\left\{\begin{array}{l}
\left(h \frac{b-a}{2}\right)^{3} \\
+\left[x-\left(b-h \frac{b-a}{2}\right)\right]^{\prime \prime} \|_{\infty}
\end{array}\right]
\end{array}\right] \frac{6(\alpha+\beta)}{6(x-2} \\
& f^{\prime \prime} \in L_{\infty}[a, b]
\end{aligned}
$$

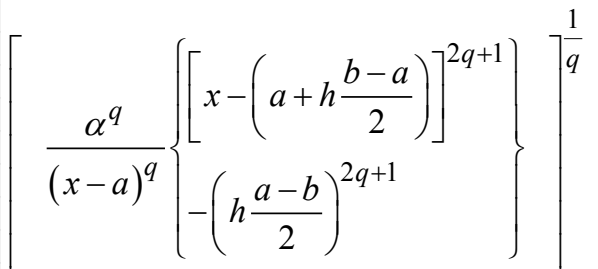

$$
\begin{aligned}
& \left.\leq\left\{\begin{array}{c}
\left(h \frac{a-b}{2}\right)^{2 q+1} \\
+\frac{\beta^{q}}{(b-x)^{q}}\left\{-\left[x-\left(b-h \frac{b-a}{2}\right)\right]^{2 q+1}\right.
\end{array}\right\}\right] \\
& \times \frac{\left\|f^{\prime \prime}\right\|_{p}}{2(2 q+1) \frac{1}{q}(\alpha+\beta)}, \\
& f^{\prime \prime} \in L_{p}[a, b] \\
& \alpha(x-a)+\beta(b-x)-h(b-a)(\alpha+\beta) \\
& +\frac{h^{2}(b-a)}{2}\left[\frac{\alpha}{x-a}+\frac{\beta}{b-x}\right] \\
& +\mid \beta(b-x)-\alpha(x-a)+h(b-a)(\alpha-\beta) \overline{4(\alpha+\beta)} \\
& +\frac{h^{2}(b-a)}{2}\left[\frac{\beta}{b-x}-\frac{\alpha}{x-a}\right] \mid \\
& , f^{\prime \prime} \in L_{1}[a, b] \text {. }
\end{aligned}
$$




\section{Main Results}

To establish our main results we first give the following essential definition and lemma.

Definition 1 We assume that the weight function (or density) $w:(a, b) \rightarrow 0, \infty)$ to be non-negative and integrable over its entire domain.

$$
\int_{a}^{b} w(t) d t<\infty
$$

and

$$
\int_{a}^{b} w(t) d t \neq 0
$$

The domain of $w$ may be finite or infinite and may vanish at the boundary point. We denote the moments

$$
\begin{gathered}
m(a, b)=\int_{a}^{b} w(t) d t, \\
N(a, b)=\int_{a}^{b} t w(t) d t, \\
\sigma(a, b)=\frac{N(a, b)}{m(a, b)} \\
n(a, b)=\int_{a}^{b} f(t) w(t) d t \\
\delta(a, b)=\frac{n(a, b)}{m(a, b)} .
\end{gathered}
$$

Let the functional $S_{w}(f ; a, b)$ be defined by

$$
S_{w}(f ; a, b)=f(x)-M_{w}(f ; a, b)
$$

where

$$
M_{w}(f ; a, b)=\frac{1}{\int_{a}^{b} w(x) d x^{a}} \int_{a}^{b} f(x) w(x) d x .
$$

The function $S_{w}(f ; a, b)$ represents the deviation of $f(x)$ from its weighted integral mean over $[a, b]$.

We start our main result with the following weighted identity which will be used to obtain bounds.

Lemma 5 Let $f:[a, b] \rightarrow \mathbb{R}$ be an absolutely continuous mapping. Denoted by $P_{w}(x,):.[a, b] \rightarrow \mathbb{R}$ the weighted peano kernel is then given by

$$
P_{w}(x, t)=\left\{\begin{array}{l}
\frac{\alpha}{\alpha+\beta} \frac{1}{m(a, x)} m(a, t), a \leq t \leq x \\
\frac{\beta}{\alpha+\beta} \frac{1}{m(x, b)} m(b, t), x<t \leq b
\end{array}\right.
$$

where $\alpha \geq 0, \beta \geq 0$ and not both zero. Then the following weighted identity

$$
\begin{aligned}
& \int_{a}^{b} P_{w}(x, t) f^{\prime}(t) d t \\
& =f(x)-\frac{1}{\alpha+\beta}[\alpha \delta(a, x)+\beta \delta(x, b)],
\end{aligned}
$$

holds.

Proof. From (15), we have

$$
\begin{gathered}
\int_{a}^{b} P_{w}(x, t) f^{\prime}(t) d t=\frac{\alpha}{\alpha+\beta} \frac{1}{m(a, x)} \int_{a}^{x}\left(\int_{a}^{t} w(u) d u\right) f^{\prime}(t) d t \\
+\frac{\beta}{\alpha+\beta} \frac{1}{m(x, b)} \int_{x}^{b}\left(\int_{b}^{t} w(u) d u\right) f^{\prime}(t) d t .
\end{gathered}
$$

By integration by parts formula, we get (16).

We now give our main result.

Theorem 2 Let $f:[a, b] \rightarrow \mathbb{R}$ be absolutely continuous mapping. Define

$$
\tau_{w}(x ; \alpha, \beta):=f(x)-\frac{1}{\alpha+\beta}\left[\begin{array}{l}
\alpha M_{w}(f ; a, x) \\
+\beta M_{w}(f ; x, b)
\end{array}\right]
$$

where $M_{w}(f ; a, b)$ is the weighted integral mean defined in (14), then

$$
\begin{aligned}
& \left|\tau_{w}(x ; \alpha, \beta)\right| \\
& \left\{\{\alpha[x-\sigma(a, x)]+\beta[-x+\sigma(x, b)]\} \frac{\left\|f^{\prime}\right\|_{\infty}}{(\alpha+\beta)}\right. \\
& , f^{\prime} \in L_{\infty}[a, b] \\
& \leq\left\{\left[\alpha^{q} \frac{(x-a)^{q+1}}{m^{q}(a, x)}+\beta^{q} \frac{(b-x)^{q+1}}{m^{q}(x, b)}\right]^{\frac{1}{q}} \frac{w(u)\left\|f^{\prime}\right\|_{p}}{(q+1)^{\frac{1}{q}}(\alpha+\beta)}\right. \\
& , f^{\prime} \in L_{p}[a, b], \\
& \max \left[\alpha \frac{m(a, t)}{m(a, x)}, \beta \frac{m(t, b)}{m(x, b)}\right] \frac{\left\|f^{\prime}\right\|_{1}}{(\alpha+\beta)}, f^{\prime} \in L_{1}[a, b] .
\end{aligned}
$$

Proof. Taking the modulus of (16), we have from (14) and (17)

$$
\left|\tau_{w}(x ; \alpha, \beta)\right|=\left|\int_{a}^{b} P_{w}(x, t) f^{\prime}(t) d t\right| \leq \int_{a}^{b}\left|P_{w}(x, t)\right|\left|f^{\prime}(t)\right| d t . \text { (19) }
$$

Thus, for $f^{\prime} \in L_{\infty}[a, b]$ (19) gives

$$
\left|\tau_{w}(x ; \alpha, \beta)\right| \leq\left\|f^{\prime}\right\|_{\infty} \int_{a}^{b}\left|P_{w}(x, t)\right| d t .
$$

Using (15) we obtain 


$$
\int_{a}^{b}\left|P_{w}(x, t)\right| d t
$$

$=\frac{\alpha}{\alpha+\beta} \frac{1}{m(a, x)} \int_{a}^{x} \int_{a}^{t} w(u) d u\left|d t+\frac{\beta}{\alpha+\beta} \frac{1}{m(x, b)} \int_{x}^{b}\right| \int_{b}^{t} w(u) d u \mid d t$

$$
=\frac{1}{\alpha+\beta}\{\alpha[x-\sigma(a, x)]+\beta[-x+\sigma(x, b)]\} .
$$

Hence the first inequality is proved.

$$
\left|\tau_{w}(x ; \alpha, \beta)\right| \leq\left\{\begin{array}{l}
\alpha[x-\sigma(a, x)] \\
+\beta[-x+\sigma(x, b)]
\end{array}\right\} \frac{\left\|f^{\prime}\right\|_{\infty}}{(\alpha+\beta)} .
$$

Further, using Hölder's integral inequality, from (19) we have for $f^{\prime} \in L_{p}[a, b]$

$$
\left|\tau_{w}(x ; \alpha, \beta)\right| \leq\left\|f^{\prime}\right\|_{p}\left(\int_{a}^{b}\left|P_{w}(x, t)\right|^{q} d t\right)^{\frac{1}{q}},
$$

where $\frac{1}{p}+\frac{1}{q}=1$ with $p>1$. Now

$$
(\alpha+\beta)\left(\int_{a}^{b}\left|P_{w}(x, t)\right|^{q} d t\right)^{\frac{1}{q}}
$$

$$
\begin{gathered}
=\left[\frac{\alpha^{q}}{m^{q}(a, x)} \int_{a}^{x}\left|\int_{a}^{t} w(u) d u\right|^{q} d t+\frac{\beta^{q}}{m^{q}(x, b)} \int_{x}^{b}\left|\int_{t}^{b} w(u) d u\right|^{q} d t\right]^{\frac{1}{q}} \\
\leq\left[\frac{(\alpha w(u))^{q}}{q+1} \frac{(x-a)^{q+1}}{m^{q}(a, x)}+\frac{(\beta w(u))^{q}}{q+1} \frac{(b-x)^{q+1}}{m^{q}(x, b)}\right]^{\frac{1}{q}} .
\end{gathered}
$$

This proves the second inequality.

$$
\begin{gathered}
|\tau(x, w ; \alpha, \beta)| \\
\leq\left[\alpha^{q} \frac{(x-a)^{q+1}}{m^{q}(a, x)}+\beta^{q} \frac{(b-x)^{q+1}}{m^{q}(x, b)}\right]^{\frac{1}{q}} \frac{w(u)\left\|f^{\prime}\right\|_{p}}{(q+1) \frac{1}{q}(\alpha+\beta)} .
\end{gathered}
$$

Finally, for $f^{\prime} \in L_{1}[a, b]$, we have from (19) and using (15)

$$
\left|\tau_{w}(x ; \alpha, \beta)\right| \leq \sup _{t \in[a, b]}\left|P_{w}(x, t)\right|\left\|f^{\prime}\right\|_{1}
$$

where

$$
\begin{aligned}
& (\alpha+\beta) \sup _{t \in[a, b]}\left|P_{w}(x, t)\right| \\
& =\max \left\{\alpha \frac{m(a, t)}{m(a, x)}, \beta \frac{m(t, b)}{m(x, b)}\right\}\left\|f^{\prime}\right\|_{1} .
\end{aligned}
$$

This completes the proof of theorem.
Remark 1 If we put $w(x)=1$, in (18), we get cerone's results (7). If we put $\alpha=\beta$ and $w(x)=1$, in (18), we get Dragomir's result (5). Similarly, for different weights, we can obtain a variety of results.

Remark 2 We may write

$$
\begin{gathered}
\alpha M_{w}(f ; a, x)+\beta M_{w}(f ; x, b) \\
=\alpha M_{w}(f ; a, x)+\frac{\beta}{m(x, b)}\left[\int_{a}^{b} f(u) w(u) d u\right. \\
\left.-\int_{a}^{x} f(u) w(u) d u\right] \\
=\alpha M_{w}(f ; a, x)-\frac{\beta}{m(x, b)} \int_{a}^{x} f(u) w(u) d u \\
+\frac{\beta}{m(x, b)} \int_{a}^{b} f(u) w(u) d u \\
=\left(\alpha+\beta-\beta \rho_{w}(x)\right) M_{w}(f ; a, x) \\
+\beta \rho_{w}(x) M_{w}(f ; a, b)
\end{gathered}
$$

where

$$
\rho_{w}(x)=\frac{m(a, b)}{m(x, b)} .
$$

Thus, from (17),

$$
=f(x)-\left[\begin{array}{c}
\tau_{w}(x ; \alpha, \beta) \\
\left(1-\frac{\beta}{\alpha+\beta} \sigma_{w}(x)\right) M_{w}(f ; a, x) \\
+\frac{\beta}{\alpha+\beta} \sigma_{w}(x) M_{w}(f ; a, b)
\end{array}\right]
$$

so that for fixed $[a, b], M_{w}(f ; a, b)$ is also fixed.

Corollary 1 If we put $\alpha=\beta$ in (18), we get

$$
\left\{\begin{array}{l}
\left\{f(x)-\frac{1}{2}\left[M_{w}(f ; a, x)+M_{w}(f ; x, b)\right] \mid\right. \\
\{\sigma(x, b)-\sigma(a, x)\} \frac{\left\|f^{\prime}\right\|_{\infty}}{2}, f^{\prime} \in L_{\infty}[a, b] \\
{\left[\frac{(x-a)^{q+1}}{m^{q}(a, x)}+\frac{(b-x)^{q+1}}{m^{q}(x, b)}\right]^{\frac{1}{q}} \frac{w(u)\left\|f^{\prime}\right\|_{p}}{2(q+1)^{\frac{1}{q}}}, f^{\prime} \in L_{p}[a, b],} \\
\max \left\{\frac{m(a, t)}{m(a, x)}, \frac{m(t, b)}{m(x, b)}\right\} \frac{\left\|f^{\prime}\right\|_{1}}{2}, f^{\prime} \in L_{1}[a, b] .
\end{array}\right.
$$

Corollary 2 Let the conditions of Theorem 2 holds. Then 


$$
\begin{aligned}
& \left|f\left(\frac{a+b}{2}\right)-\frac{1}{(\alpha+\beta)}\left[\begin{array}{l}
\alpha M\left(f, w ; a, \frac{a+b}{2}\right) \\
+\beta M\left(f, w ; \frac{a+b}{2}, b\right)
\end{array}\right]\right| \\
& \left\{\begin{array}{l}
\alpha\left[\frac{a+b}{2}-\sigma\left(a, \frac{a+b}{2}\right)\right] \\
+\beta\left[-\frac{a+b}{2}+\sigma\left(\frac{a+b}{2}, b\right)\right]
\end{array}\right\} \frac{\left\|f^{\prime}\right\|_{\infty}}{(\alpha+\beta)} \\
& , f^{\prime} \in L_{\infty}[a, b] \\
& \leq\left\{\left(\frac{b-a}{2}\right)^{q+1}\left\{\begin{array}{l}
\frac{\alpha^{q}}{m^{q}\left(a, \frac{a+b}{2}\right)} \\
+\frac{\beta^{q}}{m^{q}\left(\frac{a+b}{2}, b\right)}
\end{array}\right\}\right]^{\frac{1}{q}} \frac{w(u)\left\|f^{\prime}\right\|_{p}}{(q+1)^{\frac{1}{q}}(\alpha+\beta)} \\
& , f^{\prime} \in L_{p}[a, b], \\
& \begin{array}{l}
\max \left\{\alpha \frac{m(a, t)}{m\left(a, \frac{a+b}{2}\right)}, \beta \frac{m(t, b)}{m\left(\frac{a+b}{2}, b\right)}\right\} \frac{\left\|f^{\prime}\right\|_{1}}{(\alpha+\beta)}, \\
f^{\prime} \in L_{1}[a, b] .
\end{array}
\end{aligned}
$$

Proof. Placing $x=\frac{a+b}{2}$ in (17) and (18) produces the results stated in (22).

Corollary 3 If (21) is evaluated at the midpoint, then we get

$$
\begin{aligned}
& \left|f\left(\frac{a+b}{2}\right)-\frac{1}{2} M_{w}(f, w ; a, b)\right| \\
& \left\{\begin{array}{l}
\left.\left\{\sigma\left(\frac{a+b}{2}, b\right)-\sigma\left(a, \frac{a+b}{2}\right)\right\}\right\} \frac{f^{\prime} \|_{\infty}}{2} \\
\leq\left\{\begin{array}{l}
\left.\left(\frac{b-a}{2}\right)^{q+1}\left(\frac{1}{m^{q}\left(a, \frac{a+b}{2}\right)}\right)\right]^{\frac{1}{q}} \\
\left.+\frac{1}{m^{q}\left(\frac{a+b}{2}, b\right)}\right)
\end{array} \frac{w(u)\left\|f^{\prime}\right\|_{p}}{2(q+1)^{\frac{1}{q}}}\right.
\end{array}\right. \\
& \max \left\{\frac{m(a, t)}{m\left(a, \frac{a+b}{2}\right)}, \frac{m(t, b)}{m\left(\frac{a+b}{2}, b\right)}\right\} \frac{\left\|f^{\prime}\right\|_{1}}{2} .
\end{aligned}
$$

\section{An Application to the Cumulative Distribution Function}

Let $X$ be a random variable taking values in the finite interval $[a, b]$ with Cumulative Distributive Function

$$
F_{w}(x)=P_{r}(X \leq x)=\int_{a}^{x} f(u) w(u) d u,
$$

we also use the fact that

$$
\int_{a}^{b} f(u) w(u) d u=1,
$$

where $f$ is a Probability Density Function. The following theorem holds.

Theorem 3 Let $X$ and $F$ be as above, then

$$
\leq\left\{\begin{array}{l}
\left|\begin{array}{l}
{[\alpha m(x, b)-\beta m(a, x)] F_{w}(x)} \\
-m(a, x)[(\alpha+\beta) m(x, b) f(x)-\beta]
\end{array}\right| \\
f^{\prime} \in L_{\infty}[a, b] \\
\left.m(a, x) m(x, b)\left[\begin{array}{l}
\alpha[x-\sigma(a, x)] \\
+\beta[-x+\sigma(x, b)]
\end{array}\right\}\left\|f^{\prime}\right\|_{\infty} \frac{(x-a)^{q+1}}{m^{q}(a, x)}\right]^{\frac{1}{q}} \\
\left.+\beta^{q} \frac{(b-x)^{q+1}}{m^{q}(x, b)}\right] \frac{w(u)\left\|f^{\prime}\right\|_{p}}{(q+1)^{\frac{1}{q}}} \\
f^{\prime} \in L_{p}[a, b], \\
m(a, x) m(x, b) \max \left\{\alpha \frac{m(a, t)}{m(a, x)}, \beta \frac{m(t, b)}{m(x, b)}\right\}\left\|f^{\prime}\right\|_{1} \\
f^{\prime} \in L_{1}[a, b] .
\end{array}\right.
$$

Proof. From (18), we have

$$
\begin{gathered}
\tau_{w}(x ; \alpha, \beta) \\
=f(x)-\frac{1}{\alpha+\beta}\left[\alpha M_{w}(f ; a, x)+\beta M_{w}(f ; x, b)\right] \\
=f(x)-\frac{1}{\alpha+\beta}\left[\begin{array}{c}
\frac{\alpha}{m(a, x)} \int_{a}^{x} f(u) w(u) d u \\
+\frac{\beta}{m(x, b)} \int_{x}^{b} f(u) w(u) d u
\end{array}\right] .
\end{gathered}
$$

After simple calculations, we get

$$
\begin{aligned}
& -(\alpha+\beta) m(a, x) m(x, b) \tau_{w}(x ; \alpha, \beta) \\
= & (\alpha m(x, b)-\beta m(a, x)) F_{w}(x) \\
- & (\alpha+\beta) m(a, x) m(x, b) f(x)+\beta m(a, x)
\end{aligned}
$$




$$
\begin{aligned}
& =(\alpha m(x, b)-\beta m(a, x)) F_{w}(x) \\
& -m(a, x)[(\alpha+\beta) m(x, b) f(x)-\beta] . \\
& \left|(\alpha+\beta) m(a, x) m(x, b) \tau_{w}(x ; \alpha, \beta)\right| \\
& =\left|\begin{array}{l}
(\alpha m(x, b)-\beta m(a, x)) F_{w}(x) \\
-m(a, x)[(\alpha+\beta) m(x, b) f(x)-\beta]
\end{array}\right| .
\end{aligned}
$$

By using (18), we get (24).

Putting $\alpha=\beta=1 / 2$ in Theorem 3 gives the following result.

Corollary 4 Let $X$ be a random variable, $F_{w}(x)$ weighted Cumulative Distributive Function and $f$ is a Probability Density Function. Then

$$
\begin{aligned}
& \left|\frac{1}{2}[m(x, b)-m(a, x)] F_{w}(x)\right| \\
& -m(a, x)\left[m(x, b) f(x)-\frac{1}{2}\right] \\
& {\left[\frac{1}{2} m(a, x) m(x, b)\left\{\begin{array}{l}
\sigma(x, b) \\
-\sigma(a, x)
\end{array}\right\}\left\|f^{\prime}\right\|_{\infty}, f^{\prime} \in L_{\infty}[a, b]\right.}
\end{aligned}
$$

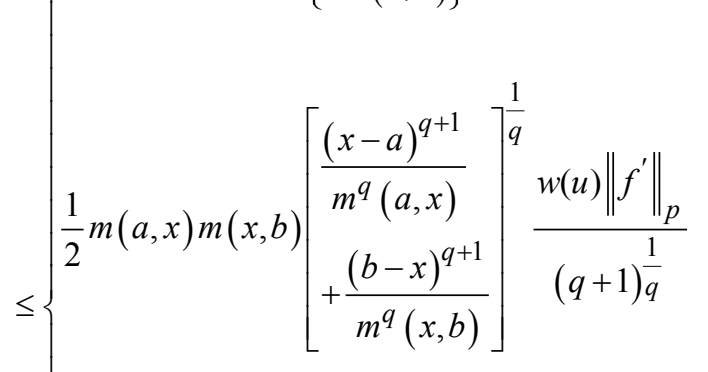

$$
\begin{aligned}
& f^{\prime} \in L_{p}[a, b], \\
& \begin{array}{l}
\frac{1}{2} m(a, x) m(x, b) \max \left\{\frac{m(a, t)}{m(a, x)}, \frac{m(t, b)}{m(x, b)}\right\}\left\|f^{\prime}\right\|_{1}, \\
f^{\prime} \in L_{1}[a, b] .
\end{array}
\end{aligned}
$$

Remark 3 The above result allow the approximation of $F_{w}(x)$ in terms of $f(x)$. The approximation of

$$
R_{w}(x)=1-F_{w}(x),
$$

could also be obtained by a simple substitution. $R_{w}(x)$ is of importance in reliability theory where $f(x)$ is the Probability Density Function of failure.

Remark 4 Put $\beta=0$ in (24) and, assuming that $\alpha \neq 0$ to obtain

$$
\leq\left\{\begin{array}{l}
m(a, x)[x-\sigma(a, x)]\left\|f^{\prime}\right\|_{\infty}, f^{\prime} \in L_{\infty}[a, b] \\
(x-a)^{1+\frac{1}{q}} \frac{w(u)\left\|f^{\prime}\right\|_{p}}{(q+1) \frac{1}{q}}, f^{\prime} \in L_{p}[a, b], \\
m(a, x)\left\|f^{\prime}\right\|_{1}, f^{\prime} \in L_{1}[a, b] .
\end{array}\right.
$$

Further we note that

$$
\begin{aligned}
& \int_{a}^{b} F_{w}(u) d u=\left.u F_{w}(u)\right|_{a} ^{b}-\int_{a}^{b} x w(x) f(x) d x \\
& =b-E[X w(X)] .
\end{aligned}
$$

\section{References}

[1] P. Cerone, A new Ostrowski type inequality involving integral Means over end intervals, Tamkang Journal Of Mathematics Volume 33, Number 2, 2002.

[2] P. Cerone and S.S. Dragomir, Trapezoidal type rules from an inequalities point of view, Handbook of Analytic-Computational Methods in Applied Mathematics, CRC Press N.Y. (2000).

[3] X. L. Cheng, Improvement of some Ostrowski-Grüss type inequalities, Comput. Math. Appl. 42 (2001), 109114.

[4] S. S. Dragomir and N. S. Barnett, An Ostrowski type inequality for mappings whose second derivatives are bounded and applications, RGMIA Research Report Collection, V.U.T., 1(1999), 67-76.

[5] S. S. Dragomir and S. Wang, A new inequality Ostrowski's type in $\mathrm{L}_{\mathrm{p}}$-norm, Indian J. of Math. 40 (1998), 299-304.

[6] S. S. Dragomir and S. Wang, A new inequality Ostrowski's type in $\mathrm{L}_{1}$-norm and applications to some special means and some numerical quadrature rules, Tamkang J. of Math. 28(1997), 239244.

[7] S. S. Dragomir and S. Wang, An inequality Ostrowski-Grüss type and its applications to the estimation of error bounds for some special means and for some numerical quadrature rules, Computers Math. Applic. 33(1997), 15-22.

[8] S. S. Dragomir and S. Wang, Applications of Ostrowski's inequality to the estimation of error bounds for some special means and to some numerical quadrature rules, Appl. Math.Lett. 11(1998), 105-109.

[9] S. S. Dragomir and S. Wang, An inequality of Ostrowski-Grüs type and its applications to the estimation of error bounds for some special means and for some numerical quadrature rules, Comput. Math. Appl., 33 (11), 15-20, (1997).

[10] Z. Liu, Some companions of an Ostrowski type inequality and application, J. Inequal. in Pure and Appl. Math, 10(2), 2009, Art. 52.

[11] G. V. Milovanovic' and J. E. Pecaric', On generalization of the inequality of A. Ostrowski and some related applications, Univ. Beograd Publ. Elektrotehn. Fak. Ser. Mat. Fiz. (544-576), 155-158, (1976).

[12] D. S. Mitrinovic', J. E. Pecaric' and A. M. Fink, Inequalities for Functions and Their Integrals and Derivatives, Kluwer Academic Publishers, 1994.

[13] A.Ostrowski, Uber die Absolutabweichung einer di erentienbaren Funktionen von ihren Integralimittelwert, Comment. Math. Hel. 10 (1938), 226-227.

[14] A. Qayyum, M. Shoaib, A. E. Matouk, and M. A. Latif, On New Generalized Ostrowski Type Integral inequalities, Abstract and Applied Analysis, Volume 2014, Article ID 275806.

[15] A. Qayyum, M. Shoaib, and I. Faye, Some New Generalized Results on Ostrowski Type Integral Inequalities With Application, Journal of computational analysis and applications, vol. 19, No.4, 2015.

[16] A. Qayyum, I. Faye, M. Shoaib, M.A. Latif, A Generalization of ostrowski type inequality for mappings whose second derivatives belong to $\mathrm{L}_{1}(\mathrm{a}, \mathrm{b})$ and applications, International Journal of Pure and Applied Mathematics, 98 (2) 2015, 169-180.

[17] A. Qayyum, M. Shoaib, M.A. Latif, A Generalized inequality of Ostrowski type for twice differentiable bounded mappings and applications, Applied Mathematical Sciences, Vol. 8, 2014, (38), 1889-1901. 